Purdue University

Purdue e-Pubs

CTRC Research Publications

Cooling Technologies Research Center

2015

\title{
Performance-Governing Transport Mechanisms for Heat Pipes at Ultra-thin Form Factors
}

Y. Yadavalli

Purdue University

J.A. Weibel

Purdue University, jaweibel@purdue.edu

S V. Garimella

Purdue University, sureshg@purdue.edu

Follow this and additional works at: http://docs.lib.purdue.edu/coolingpubs

Yadavalli, Y.; Weibel, J. A.; and Garimella, S V., "Performance-Governing Transport Mechanisms for Heat Pipes at Ultra-thin Form Factors" (2015). CTRC Research Publications. Paper 286.

http://dx.doi.org/10.1109/TCPMT.2015.2477275

This document has been made available through Purdue e-Pubs, a service of the Purdue University Libraries. Please contact epubs@purdue.edu for additional information. 


\title{
Performance-Governing Transport Mechanisms for Heat Pipes at Ultra-Thin Form Factors
}

\author{
Yashwanth Yadavalli, Justin A. Weibel, and Suresh V. Garimella
}

\begin{abstract}
Heat pipes and vapor chamber heat spreaders offer a potential solution to the increasing thermal management challenges in thin-form-factor mobile computing platforms, where efficient spreading is required to simultaneously prevent overheating of internal components and formation of hot regions on the device exterior surfaces. Heat pipe performance limitations unique to such ultra-thin form factors, and the key heat transfer mechanisms governing the performance, must be characterized. A thermal resistance network model and a detailed two-dimensional numerical model are used to analyze the performance of heat pipes under these conditions. A broad parametric study of geometries and heat inputs using the reduced-order model helps delineate the performance thresholds within which the effectiveness of a heat pipe is greater than a comparable solid heat spreader. A vapor-phase threshold unique to ultra-thin heat pipes operating at low power inputs is observed. At this threshold, the vapor-phase thermal resistance imposed by the saturation pressure/temperature gradient in the heat pipe causes a crossover in the thermal resistance relative to a solid heat spreader. The higher-fidelity numerical model is used to assess the accuracy of the resistance network model and to verify the validity and applicability of each assumption made regarding the transport mechanisms. Key heat transfer mechanisms not captured by the reduced-order thermal network models are identified. These include the effects of boundary conditions on the interface mass flux profile, convective effects on the vapor core temperature drop, and two-dimensional conduction on smearing of evaporation/condensation mass flux into the adiabatic section.
\end{abstract}

Index Terms - flat heat pipe, low heat flux, numerical model, performance limits, ultra-thin vapor chamber

\section{NOMENCLATURE}

A area, $\mathrm{m}^{2}$

$C$ specific heat capacity, $\mathrm{J} / \mathrm{kgK}$

$C_{E}$ Ergun's coefficient

$h_{f g}$ latent heat, $\mathrm{J} / \mathrm{kg}$

$k$ thermal conductivity, $\mathrm{W} / \mathrm{mK}$

$K$ permeability, $\mathrm{m}^{2}$

$L$ length, $m$

$m$ mass flow rate, $\mathrm{kg} / \mathrm{s}$

$m^{\prime \prime}$ mass flux, $\mathrm{kg} / \mathrm{m}^{2} \mathrm{~s}$

$M$ figure of merit $\left[\left(\rho_{l} \sigma_{l} L\right) / \mu_{l}\right]$

$M_{v}$ vapor figure of merit $\left[\left(h_{f g}^{2} \rho_{v} P_{v}\right) /\left(\mathbf{R} \mu_{v} T_{v}^{2}\right)\right]$

$q^{\prime \prime}$ heat flux, $\mathrm{W} / \mathrm{m}^{2}$

$P$ pressure, $\mathrm{Pa}$

$P_{\text {cap }}$ capillary pressure, $\mathrm{Pa}$

$P_{v} \quad$ saturated vapor pressure, $\mathrm{Pa}$

$R$ thermal resistance, $\mathrm{K} / \mathrm{W}$

$\mathrm{R}$ gas constant, $\mathrm{J} / \mathrm{kgK}$

$r$ thickness ratio

$t$ thickness, $\mathrm{m}$

$t_{\text {limit }}$ limiting thickness, $\mathrm{m}$

$T$ temperature, $\mathrm{K}$

$u \quad x$-direction velocity, $\mathrm{m} / \mathrm{s}$

$v$ y-direction velocity, $\mathrm{m} / \mathrm{s}$ $x$ axial coordinate, $\mathrm{m}$

$y$ transverse coordinate, $\mathrm{m}$

\section{GREEK SYMBOLS}

$\rho$ density, $\mathrm{kg} / \mathrm{m}^{3}$

$\varphi$ porosity

$\mu$ dynamic viscosity, $\mathrm{N} / \mathrm{m}$

$\sigma$ surface tension, $\mathrm{Ns} / \mathrm{m}^{2}$

$\hat{\sigma}$ accommodation coefficient

SUBSCRIPTS

0 reference

$a$ adiabatic

$c$ condenser

$e$ evaporator

eff effective

ext external

$i$ interface

$v$ vapor

$l$ liquid

$s$ solid

wl wall

$w$ wick

HP heat pipe

$H S$ heat spreader 


\section{INTRODUCTION}

A S mobile computing devices become thinner and more powerful, efficient heat spreading technologies with matched ultrathin form factors are called for. Heat pipes, which can have effective conductivities that are orders of magnitude larger than the intrinsic thermal conductivity of solid materials, are a viable alternative; however, demand for device thicknesses less than 1 $\mathrm{mm}$ imposes both practical fabrication challenges and potential performance limitations. Heat sources generating less than $1 \mathrm{~W}$ in these target applications are outside typical heat pipe design objectives, and recent studies have focused on increasing the maximum heat dissipation while simultaneously reducing thickness [1], as opposed to minimizing the thermal resistance at low heat fluxes. The operating conditions of ultra-thin heat pipes at low input heat fluxes brings unique performance requirements to the fore, especially in regard to user comfort that would not otherwise be central. This represents a paradigm shift in thermal management requirements, where handheld devices must maintain external surface temperatures driven by user comfort. This is often the limiting factor that determines performance throttling, rather than peak junction temperatures in the system on chip (SoC). Mobile devices also have numerous other components which may be temperature-limited (e.g., the display and battery) or may dwarf the SoC heat generation under certain usage conditions (e.g., the modem). This new regime of operating conditions gives rise to unique performance limitations in heat pipes; this paper analyses these limitations using models to simulate transport in heat pipes.

Many analytical and numerical modeling approaches have been developed to study the performance of heat pipes at varying levels of spatial and temporal fidelity, as reviewed for various types of heat pipes by Garimella and Sobhan [2] and Faghri [3]. A simplified thermal resistance network-based analysis of heat pipe performance by Prasher [4] accurately predicted thermal resistance even under the nonuniform experimental heating conditions investigated by Chang et al. [5]. Vafai and Wang [6] first proposed a pseudo-three-dimensional analytical model that solved for the steady-state velocity field and axial temperature distribution assuming incompressible fluid flow; the approach was extended to include transient start-up and shutdown [7]. Aghvami and Faghri [8] developed an analytical model for prediction of the two-dimensional temperature distribution in a heat pipe and analyzed various heating and cooling configurations. Koito et al. [9] and Xiao and Faghri [10] developed threedimensional, steady-state numerical models which solved for heat transport and fluid flow in the wall, vapor core, and porous wick domains. Vadakkan et al. [11, 12] developed a three-dimensional numerical model to study the transient performance of heat pipes with multiple discrete heat input sources. Ranjan et al. [13] coupled this device-scale numerical model with a microscale model that captured the vapor-liquid interface curvature effects on evaporation/condensation. Famouri et al. [14] recently performed a 2D numerical analysis of heat pipe operation implementing the transient volume-averaged gas density treatment developed by $[11,12]$ and proposed a selective under-relaxation method for mass transfer across the interface.

Analytical and numerical models typically do not capture other phenomena that may result in operational limits to the maximum heat transfer capacity of a heat pipe, such as entrainment of liquid into the vapor phase by shearing of the interface, local dryout at the evaporator due to boiling, and choking of the vapor flow at the sonic limit [15]. A capillary limit is commonly encountered when the pressure head provided by the wick structure at the evaporator cannot support the cumulative fluid pressure drop throughout the capillary-driven loop; vapor pressure drop is typically neglected, and the capillary limit is dictated by a working fluid figure of merit $M$ which captures relevant liquid-phase properties. These transport-limiting phenomena prevent normal operation of the device; however, other practical design thresholds may arise when the performance of a heat pipe is unfavorable compared to a solid heat spreader under certain operating conditions and geometries. These thresholds are not fundamental limits to the maximum/minimum heat flux, but instead provide useful insight into the practicality of employing heat pipes. Sauciuc et al. [16] investigated the ratio of thermal resistance imposed by a flat heat pipe to that of solid copper serving as the heat spreading base of an air-cooled fin heat sink. A threshold was identified with increasing heat spreader thickness where solid copper becomes favored over the heat pipe. Device performance thresholds with decreasing thicknesses of less than $1 \mathrm{~mm}$ were not investigated over the range of heat input investigated $\left(10-50 \mathrm{~W} / \mathrm{cm}^{2}\right)$.

This paper identifies the important heat transfer mechanisms and practical performance thresholds that need to be taken into consideration when designing ultra-thin form factor heat pipes operating at low input heat fluxes. Using a reduced-order thermal resistance network model, a broad parametric study is conducted to assess performance as a function of geometry and heat input for a canonical heat pipe configuration. Performance is benchmarked against a solid heat spreader to define the practical performance thresholds. A subsequent comparison against the performance predicted by a higher-fidelity numerical model allows assessment of the various simplifying assumptions employed in the thermal resistance network model, thereby identifying the critical heat transfer mechanisms that may not be accounted for in a reduced-order model under these operating conditions.

\section{MOdeling ApPROACH}

The flat heat pipe configuration considered in the current study is shown in Fig. 1(a). The working fluid is vaporized at the wick-vapor interface as heat is applied to the evaporator section; as a result of the local pressure increase, vapor flows to the condenser end. Heat removal at the condenser causes the vapor to condense into the saturated porous wick structure, and capillary pressure pumps liquid back to the evaporator. Within the heat pipe, the wick structure lines the same side of the heat 
pipe as the evaporator and condenser sections to which heating and cooling boundary conditions are respectively applied. All other external walls of the heat pipe are considered to be adiabatic.

\section{A. Thermal Resistance Network Model}

The temperature drop due to the primary heat transport mechanisms that occur in each section of the heat pipe can be represented using the simplified effective thermal resistance network shown in Fig. 1(b). The thermal resistances due to conduction through the thickness of the wall in the evaporator $\left(R_{w l, e}\right)$ and condenser $\left(R_{w l, c}\right)$ sections are negligible in the current study, which is targeted at ultra-thin heat pipes constructed from high-conductivity materials. Lateral conduction along the heat pipe wall is shown as $R_{w l, \text { lateral }}$; this thermal resistance can be calculated using an effective device length that takes into account the varying heat load along the length of the evaporator and condenser sections, given by

$$
L_{\text {eff }}=L_{a}+\frac{L_{e}+L_{c}}{2}
$$

Resistances through the wick in the evaporator $\left(R_{w, e}\right)$, condenser $\left(R_{w, c}\right)$, and laterally across the wick ( $\left.R_{w, l a t e r a l}\right)$, are assumed to be due to conduction only. Convective heat transfer in the porous medium is neglected due to the small interstitial liquid velocities; hence, the liquid flow field need not be solved. An effective saturated wick thermal conductivity is used to estimate these one-dimensional conduction resistances. Lateral heat flow through the wick is neglected, owing to the high resistance that results from the low thermal conductivity and high aspect ratio wick geometry.

The interfacial phase-change thermal resistances at the wick-vapor interface in the evaporator and condenser sections, represented by $R_{i, e}$ and $R_{i, c}$, respectively, are typically small [15] and therefore neglected. The effective thermal resistance of the vapor core $R_{v}$ is calculated by simplifying the vapor flow field as fully developed flow between parallel plates to estimate the pressure drop in the vapor core over the effective length. This pressure drop is related to the saturation temperature drop by applying the Clapeyron equation and ideal gas law. By this approach, an effective thermal conductivity [4] is defined for a onedimensional lateral resistance along the effective length of the vapor

$$
k_{e f f, v}=\frac{h_{f g}^{2} P_{v} \rho_{v} t_{v}^{2}}{12 \mathbf{R} \mu_{v} T_{v}^{2}}
$$

where the thermophysical properties are those of saturated vapor and are evaluated at the local vapor temperature that varies along the effective length of the device. To implement temperature-dependent vapor properties, the one-dimensional vapor thermal resistance is discretized, and each individual resistance is back-calculated from the known condenser-side temperature, and by iterating upon the heat flux passing through the vapor core. This approach does not consider convection effects within the vapor core.

Combining the system resistances yields the effective heat pipe resistance

$$
R_{H P}=\frac{R_{w l, \text { lateral }}\left(R_{v}+R_{w, e}+R_{w, c}\right)}{R_{w l, \text { lateral }}+R_{v}+R_{w, e}+R_{w, c}}
$$

An external heat sink resistance ( $R_{\text {ext }}$ ) is included on the condenser side to replicate the realistic coupling between the ambient temperature and the heat pipe operating temperature as a function of heat load.

The model is implemented as a set of simultaneous non-linear equations in Engineering Equation Solver (EES) [17], which includes temperature-dependent thermophysical vapor properties.

\section{B. 2 D Numerical Model}

The numerical model employed here is adapted from [11]. In summary, the model solves for the continuity, momentum, and energy equations in the wall, wick, and vapor regions of the heat pipe. Evaporation and condensation at the interface is determined from a kinetic theory-based formulation that uses the interface temperature determined from a local energy balance. To accommodate the transient changes in the mass of vapor, the vapor density is calculated from the operating pressure and local temperature using the ideal gas law; the volume-averaged liquid density is adjusted to conserve mass. All other thermophysical properties are assumed to be constant.

The liquid and vapor flow are considered to be laminar and incompressible. The continuity equation is as follows

$$
\varphi \frac{\partial \rho}{\partial t}+\nabla \cdot(\rho V)=0
$$

The term $\partial \rho / \partial t$ accounts for mass addition or depletion in the fluid. The momentum equations with a Brinkman-Forchheimer extended Darcy model for fluid flow in the wick region and the vapor core are 


$$
\begin{aligned}
& \frac{\partial \rho u}{\partial t}+\nabla \cdot(\rho V u)=-\frac{\partial \varphi p}{\partial x}+\nabla \cdot(\mu \nabla u)-\frac{\mu \varphi}{K} u-\frac{C_{E} \varphi}{\sqrt{K}} \rho|V| u \\
& \frac{\partial \rho v}{\partial t}+\nabla \cdot(\rho V v)=-\frac{\partial \varphi p}{\partial y}+\nabla \cdot(\mu \nabla v)-\frac{\mu \varphi}{K} v-\frac{C_{E} \varphi}{\sqrt{K}} \rho|V| v
\end{aligned}
$$

In the vapor core, permeability $K=\infty$ and porosity $\varphi=1$. The energy equation in the wall, wick, and vapor core is

$$
\frac{\partial(\rho C)_{m} T}{\partial t}+\nabla \cdot\left[(\rho C)_{l} V T_{l}\right]=\nabla \cdot\left(k_{e f f} \nabla T\right)
$$

where $(\rho C)_{m}$ assumes different values in the wall, wick, and vapor core:

$$
\begin{aligned}
& \text { Wall : }(\rho C)_{m}=(\rho C)_{s} \\
& \text { Wick : }(\rho C)_{m}=(1-\varphi)(\rho C)_{s}+\varphi(\rho C)_{l} \\
& \text { Vapor core }:(\rho C)_{m}=(\rho C)_{v}
\end{aligned}
$$

The effective conductivity $k_{\text {eff }}$ also assumes appropriate values in the wall, wick, and vapor core. The following boundary conditions are imposed on the domain:

1. Wick-vapor core interface: Liquid-vapor phase change is assumed to occur at the wick-vapor core interface. The interface temperature $T_{i}$ is obtained from an energy balance at the interface accounting for conduction and convection on the liquid and vapor sides, and phase change

$$
-k_{\text {wick }} A_{i} \frac{\partial T}{\partial y}+m_{i} C_{l} T_{i}=-k_{v} A_{i} \frac{\partial T}{\partial y}+m_{i} C_{l} T_{i}+m_{i} h_{f g}
$$

Here $m_{i}<0$ denotes evaporation and $m_{i}>0$ denotes condensation. The interface pressure $\boldsymbol{P}_{\boldsymbol{i}}$ is obtained from the Clausius-Clapeyron equation with $P_{0}$ and $T_{0}$ being reference values

$$
\frac{\mathbf{R}}{h_{f g}} \ln \left(\frac{P_{i}}{P_{0}}\right)=\frac{1}{T_{0}}-\frac{1}{T i}
$$

The interface mass flux is calculated using the kinetic theory-based interfacial transport expression proposed by Schrage [18]

$$
\left(\frac{2 \hat{\sigma}}{2-\hat{\sigma}}\right)\left(\frac{1}{\sqrt{2 \pi \mathbf{R}}}\right)\left(\frac{P_{v}}{\sqrt{T_{v}}}-\frac{P_{i}}{\sqrt{T_{i}}}\right)=m_{i}^{\prime \prime}
$$

A value of 0.03 is chosen for the accommodation coefficient $\hat{\sigma}$ as a lower bound for water [13]. The evaporated and condensed mass is assumed to flow in a direction normal to the interface when accounting for momentum transport due to phase change.

2. Wick-wall interface:

$$
u=0, v=0
$$

3. Upper wall: The evaporator section has a constant heat flux condition

In the adiabatic section

$$
k_{w} \frac{\partial T}{\partial y}=q_{e} ; 0 \leq x \leq L_{e}
$$

$$
\frac{\partial T}{\partial y}=0 ; u=v=0 ; L_{e} \leq x \leq L_{e}+L_{a}
$$

The condenser section has a convection condition with heat transfer coefficient $h_{C}$ and ambient temperature $T_{C}$

$$
-k_{w} \frac{\partial T}{\partial y}=h_{c}\left(T-T_{c}\right) ; x>L_{e}+L_{a}
$$

4. Lateral walls:

$$
u=v=\partial T / \partial y=0
$$

5. Bottom wall:

$$
u=v=\partial T / \partial x=0
$$

In addition, the following initial conditions are imposed:

$$
T(x, y, 0)=T_{i} ; P_{o p}(t=0)=P_{\text {sat }}\left(T_{i}\right)
$$


To improve the stability of the model, and account for the coupling of interface mass flow with temperature, the mass flow rate is linearized with respect to temperature. To prevent round-off errors in the computation of pressure gradients from pressure differences that are small compared to the absolute pressure, the pressure is split into two components: a system operating pressure and a hydrodynamic component. These implementation details are discussed further in [11, 12].

The transient governing equations are discretized using the finite volume method and solved with the commercial solver FLUENT [19]. The rectangular mesh elements have a constant aspect ratio of 5 for all cases, with a size of either $0.04 \mathrm{~mm} \times 0.20$ $\mathrm{mm}$ or $0.05 \mathrm{~mm} \times 0.25 \mathrm{~mm}$ to achieve a uniform grid over the domain for the different heat pipe geometries investigated. The wick-vapor interface conditions, evaporation and condensation mass flow rates, and fluid densities are computed with userdefined functions. In this work, steady state is considered to be achieved when the heat transfer rate in the condenser section reaches within $0.1 \%$ of the value at the evaporator section. The solution at each time step is considered converged when the residuals are less than $10^{-6}$ for the continuity and momentum equations, and less than $10^{-10}$ for the energy equation.

\section{RESULTS AND DISCUSSION}

The thermal resistance network model allows initial performance evaluation for screening a large number of geometric permutations to identify the performance limits. Some device geometry parameters and operating conditions are fixed to specific values to highlight the important performance limits that occur at small device thicknesses and low input powers. For the configuration shown in Fig. 1(a), the length of the evaporator and condenser sections are both held constant at $10 \mathrm{~mm}$, while the length of the adiabatic section and the total thickness are varied. The relative thickness of each region is fixed; the walls are $10 \%$ of the total thickness and the wick and vapor core are each $40 \%$ of the total thickness. The range of geometries investigated (10 $\mathrm{mm}<L_{a}<100 \mathrm{~mm} ; 0.2 \mathrm{~mm}<t<20 \mathrm{~mm}$ ) is intended to establish theoretical limits at extremes, and is not necessarily indicative of fabrication or material constraints. Over the range of the geometries investigated, lateral conduction resistances in the wick were found to be an order of magnitude larger than the vapor core resistance.

The heat pipe wall material is copper with a thermal conductivity of $400 \mathrm{~W} / \mathrm{mK}$. The wick consists of sintered copper powder with particle sizes in the range $45-75 \mu \mathrm{m}$; this particle size range allows the formation of the thin wick layers needed. The effective properties for this wick structure were characterized via x-ray microtomography-based simulations by Bodla et al. [20]. The wick effective thermal conductivity is $55.7 \mathrm{~W} / \mathrm{m} \mathrm{K}$, its permeability is $1.43 \times 10^{-11} \mathrm{~m}^{2}\left(C_{E}=0.55\right)$ and it supports a capillary pressure of $2250 \mathrm{~Pa}$. A constant heat flux is applied to the evaporator section, and the condenser section has a convective heat transfer coefficient of $2604 \mathrm{~W} / \mathrm{m}^{2} \mathrm{~K}$ with an ambient temperature of $287 \mathrm{~K}$.

The net resistance of the heat pipe is calculated and benchmarked against the resistance of a solid copper heat spreader of the same outer dimensions. The heat spreader resistance is calculated as a one-dimensional resistance along the effective length, which accurately accounts for the effects of two-dimensional spreading near the evaporator and condenser, as confirmed by numerical simulation. When the resistance ratio $R_{H P} / R_{H S}$ is greater than unity, the performance of the heat pipe is worse than a solid heat spreader of the same dimensions, and the condition when $R_{H P} / R_{H S}=1$ is identified as a performance threshold. The onset of a capillary limitation is also monitored, as the heat pipe may be susceptible to this particular transport limit over the range of geometries and boundary conditions investigated. The capillary limit is enforced when

$$
\Delta P_{\text {cap }, \max } \leq \Delta P_{l}+\Delta P_{v}
$$

where the pressure drop in the vapor core $\Delta P_{v}$ is calculated assuming fully developed parallel plate flow. This physical transport limitation arrests fluid flow in the device and $R_{H P} / R_{H S}$ is then assumed $>1$.

\section{A. Performance-Limiting Conditions}

By comparing the performance across different input heat fluxes and for a range of geometries, it is possible to identify the limiting conditions that become predominant at small thickness and low input power operation. Fig. 2 shows a contour map of the ratio between the heat pipe and copper heat spreader thermal resistances with the adiabatic length and total thickness being varied. The dark shaded region indicates advantageous heat pipe performance, and fades to white as the ratio transitions in favor of the copper heat spreader. The performance thresholds and the capillary limit are also indicated.

Fig. 2(a) shows the resistance ratio contour plot for a relatively high input heat flux of $2 \mathrm{~W} / \mathrm{cm}^{2}$ to illustrate the limits encountered when designing ultra-thin heat pipes for moderate power dissipation. In this case, as the thickness is reduced at a given length, the minimum thickness for which $R_{H P} / R_{H S}$ remains greater than unity is governed by the capillary limit; a sharp transition from black to white indicates this physical transport limit. At small thicknesses and relatively high heat fluxes, the mass flow is high enough that the pressure drop along the wick and vapor core is higher than the available capillary pressure. Pressure drop in the wick is an order of magnitude larger than that across the vapor core under the conditions investigated; hence, the capillary limit-based figure of merit $M$ is an appropriate design metric. In the opposite direction, with increasing device thickness, relative performance worsens for comparatively shorter and thicker heat pipes. The performance advantage of a heat pipe comes from the small resistances incurred due to phase change and the vapor core between the evaporator and condenser 
ends when heat is applied to the wick structure. This advantage is lost for short, thick heat pipes, where the effective length is reduced and solid heat spreaders provide a more direct and higher-conductivity heat flow path than the heat pipe wick. This threshold at high thickness-to-length ratios has been previously acknowledged as a design guideline in the literature [16], but is not a concern for the ultra-thin devices of interest in the current study.

Fig. 2(b) shows the limiting conditions for the case of a much lower input heat flux of $0.2 \mathrm{~W} / \mathrm{cm}^{2}$. At low input heat fluxes, there is sufficient capillary pressure due to the reduced fluid velocities, and the capillary limit shifts dramatically to lower thicknesses and higher working lengths. Instead, a new threshold appears where the resistance ratio gradually favors the solid heat spreader with decreasing thickness, despite avoidance of the capillary limit. This threshold is due to the increasing pressure drop (and corresponding saturation temperature drop) along the vapor core, and therefore is identified as a vapor resistance threshold in the figure. This threshold governs the smallest device thickness at which heat pipes are beneficial, and therefore is an important limiting condition to consider for ultra-thin heat pipes operating with low heat flux input.

Despite the specific conditions for which the vapor resistance threshold is identified in Fig. 2(b), other important generalized conclusions may be drawn. Considering that the capillary limit is not easily reached at very low input powers, and the threshold is governed by vapor phase pressure drop, a majority of the heat pipe cross-sectional area should be reserved for the vapor core. A theoretical limit to the vapor resistance threshold can be predicted by allotting the entire cross-section to the vapor core using the analysis presented next.

It is desirable to formulate a generalized expression for the limiting thickness (on the low end) governed by the vapor resistance threshold, independent of the specific geometric constraints imposed in Fig. 2(b). The total thermal resistance of the heat pipe is analytically compared to the resistance of a heat spreader under the constraint

$$
\frac{R_{H P}}{R_{H S}}<1
$$

By substituting the individual thermal resistance components and representing the thicknesses of the vapor core, wick, and walls as fractions of the total thickness, i.e., $t_{v}=r_{v} t, t_{w}=r_{w} t$, and $t_{w l}=r_{w l} t$, the threshold conditions may be expressed as

$$
\frac{r_{w}}{k_{w}}\left(\frac{1}{L_{e}}+\frac{1}{L_{c}}\right) t^{4}-\frac{L_{e f f}}{k_{s}} \frac{1}{1-r_{w}} t^{2}+\frac{L_{e f f}}{M_{v} r_{v}^{3}}=0
$$

Solving the equation results in two positive roots representing the thickness at both threshold conditions (vapor resistance and high thickness-to-length ratio). The limiting minimum thickness governed by the vapor resistance threshold is

$$
t_{\text {limit }}=\left(\frac{\frac{L_{\text {eff }}}{k_{s}} a-\sqrt{\left(\frac{L_{\text {eff }}}{k_{s}} a\right)^{2}-4 \frac{r_{w}}{k_{w}}\left(\frac{1}{L_{e}}+\frac{1}{L_{c}}\right)\left(\frac{L_{\text {eff }}}{M_{v} r_{v}^{3}}\right)}}{2 \frac{r_{w}}{k_{w}}\left(\frac{1}{L_{e}}+\frac{1}{L_{c}}\right)}\right)^{\frac{1}{2}}
$$

where $a=1 /\left(1-r_{w l}\right)$ and $M_{v}$ is a constant representing the vapor properties. This limiting thickness is independent of the heat input (except for the influence on the vapor properties with increasing temperature for fixed condenser temperature operation); however, the capillary limit should be evaluated in conjunction with this threshold, which would be expected to prevail at any moderate heat inputs. The vapor properties dictating the vapor resistance threshold are represented as a single factor that can be used as a merit number for fluid selection in the design of the ultra-thin heat pipes operating at low heat inputs

$$
M_{v}=\frac{h_{f g}^{2} \rho_{v} P_{v}}{\mathbf{R} \mu_{v} T_{v}^{2}}
$$

which is a combination of properties contained in the effective vapor conductivity [4], but presented in a form that excludes geometric parameters. The vapor figure of merit increases monotonically with temperature (primarily due to the strong temperature-dependence of vapor pressure and vapor density), indicative of improved performance and a reduced limiting thickness at higher temperatures.

\section{B. Assessment of Thermal Resistance Network Model Accuracy}

The above formulations hinge on the accuracy of the thermal resistance network model. The various assumptions made by the model are assessed, and provide further understanding of the prominent heat transfer mechanisms near these performance thresholds. A comparison of the heat pipe performance as predicted by the thermal resistance network model and the higherfidelity 2D numerical model is shown in Fig 3. The heat pipe thicknesses investigated with the numerical model $(0.4 \mathrm{~mm}, 0.5$ $\mathrm{mm}, 0.6 \mathrm{~mm}, 0.8 \mathrm{~mm}$, and $1 \mathrm{~mm}$ ) are selected to span the transition toward the vapor-resistance threshold on the basis of the 
thermal resistance network model prediction for a device length of $40 \mathrm{~mm}$. The heat pipe configuration, solid/fluid properties, geometric constraints, and boundary conditions are identical to those presented for the thermal resistance modeling results; the input heat flux at the evaporator is $0.2 \mathrm{~W} / \mathrm{cm}^{2}$. The predictions are in reasonable agreement, with the reduced-order model predicting a lower resistance than the numerical model at small thickness and higher values at larger thickness. The differences observed at both smaller and larger thicknesses are investigated further to identify the mechanistic differences.

\section{1) Heat transfer in the wick region}

The thermal resistance network model assumes the effective resistance of the wick in the evaporator and condenser sections to be only due to conduction, and ignores advection in the wick. To verify the validity of this assumption the temperature drop across the wick predicted by the resistance network and 2D numerical model are compared in the evaporator and condenser sections as shown in Table I. There is a minor difference $(<15 \%)$ in temperature drops predicted by both models, validating the assumption regarding the mode of heat transport in the wick region for this case. Further, the magnitude of the temperature drop is small and does not account for the discrepancy in the overall thermal resistance between the two modeling approaches under the conditions investigated. In general, the accuracy of this assumption can be assessed by scaling the diffusive versus advective transport based on the liquid velocity in the wick structure. The Peclet number for the current cases investigated was on the order of $10^{-5}$, which suggests that advection can be neglected as shown.

\section{2) Pressure drop across the vapor core}

The pressure drop predicted by the thermal resistance network model assumes fully developed flow between parallel plates in the adiabatic section of the vapor core. Fig. 4(a) shows the gauge pressure contours and streamtraces as predicted by the numerical model $\left(t=0.5 \mathrm{~mm}, L_{a}=20 \mathrm{~mm}\right.$ ). Fig. 4(b) compares the velocities at the start, center, and end of the adiabatic section to a fully developed velocity profile obtained using the pressure drop across the adiabatic section from the numerical model. Even though the maximum velocity magnitude varies slightly along the length of the adiabatic section, the fully developed flow profile is shown to be a good approximation.

In the evaporator and condenser sections the vapor must turn at a right angle, and the flow profile is clearly not represented by a one-dimensional, fully developed flow field. The thermal resistance network therefore assumes an effective length for which the pressure drop calculated using a fully developed flow assumption is equivalent to the actual pressure drop. The effective length used to calculate the pressure drop is based on the assumption that the rate of change of mass flux along the vapor core is constant [15]. To accurately calculate the pressure drop using this flow assumption, the mass flow rate in the vapor core calculated from the 2D numerical model has been used. A section-wise comparison of pressure drop is presented in Table II. In the adiabatic section, the calculated pressure drops match as expected from interrogation of the velocity profiles in Fig. 4(b). Minor differences can be attributed to the imprecision in evaluation of the temperature-dependent vapor properties.

In the sections where an effective length is used to represent the pressure drop, the accuracy of the calculations differs significantly; the calculated values match in the evaporator section and differ by as much as $14 \%$ in the condenser section. This is due to the different external boundary conditions imposed on these sections that result in different flow patterns. Fig. 5 shows the mass flux along the wick-vapor interface for the different thicknesses. While the constant heat flux boundary condition in the evaporator section results in an approximately flat mass flux (as assumed in the effective length used), this assumption is poor in the condenser section where the constant heat transfer coefficient boundary condition is imposed. As the thickness of the device decreases, and the interface mass flux is more closely interrelated with the external boundary conditions, the discrepancy between the actual pressure drop and that predicted using the effective length worsens.

\section{3) Temperature drop across the vapor core}

In the thermal resistance network model, the temperature drop across the vapor core solely accounts for the change in saturation temperature as calculated using the pressure drop and Clausius-Clapeyron relation

$$
\Delta T=\frac{R T^{2}}{p_{v} h_{f g}} \Delta P
$$

Table III shows a comparison between the temperature drop in the adiabatic section predicted using the full numerical model, and the saturation temperature change calculated using the Clausius-Clapeyron relation and pressure drop from the numerical model result. The Clausius-Clapeyron relation over-predicts the temperature drop compared to the numerical model at the smallest $(0.4 \mathrm{~mm})$ thickness investigated due to the neglect of convective effects. This trend changes as the thickness is increased, and switches to an under-prediction.

To understand the reason for the under-prediction of temperature drop at larger thicknesses, Fig. 6 shows a comparison of the temperature variations along the centerline of the adiabatic section between the numerical model and the Clausius-Clapeyron relation. The temperature gradients are in fair agreement at the center of the adiabatic section where there is not any mass flux into or out of the vapor core (Fig. 5). While the temperature gradient is in agreement at the center of the adiabatic section, there 
is significant deviation at either ends of the section. This disagreement can be explained by observing the spreading of heat, and thereby mass flux, into the vapor core that occurs at larger thicknesses, as can be observed in Fig. 5. As parts of the adiabatic section contribute to evaporation and condensation at either end, the temperature drop calculated by implementing the ClausiusClapeyron relation, which does not account for the addition of mass flux into the control volume when relating the temperature drop with pressure drop, is no longer accurate.

The thermal resistance network model is remarkably accurate over the range of device thicknesses investigated considering the numerous simplifying assumptions employed; however, the comparison between the 2D numerical model and the thermal resistance network model reveals several drawbacks when such simplifications are made. The mass flux profile at the wick-vapor interface is significantly influenced by the boundary conditions in the evaporator and condenser sections, which invalidates the conventional effective length used for pressure drop calculations. Convective effects in the vapor core reduce the temperature drop at very small thicknesses, and spreading of the evaporation/condensation mass flux into the adiabatic section increases the temperature drop at larger thicknesses.

\section{CONCLUSIONS}

The performance of ultra-thin form factor heat pipes was simulated using a thermal resistance network model and a higherfidelity numerical model. Using the thermal resistance network model, it was shown that the limiting smallest heat pipe thickness is defined by a vapor resistance threshold at low input heat fluxes. At this threshold, pressure drop in the vapor core causes a higher effective thermal resistance compared to a solid heat spreader. An analytical evaluation of this threshold revealed a relationship between the limiting thickness and the governing vapor properties, which is captured by defining a vapor-phase figure of merit as a criterion for working fluid selection. A detailed comparison between the numerical model and the thermal resistance network model was presented, and each of the simplifying assumptions made by the reduced-order model analyzed. The thermal resistance network, though remarkably accurate given its simplicity, fails to capture several key heat transfer mechanisms that may affect the performance of ultra-thin heat pipes. These include the effects of external boundary conditions on the interfacial mass flux profile, convection in the vapor core, and smearing of the interfacial mass flux into the adiabatic section by two-dimensional conduction. The thermal resistance network model is able to capture the transport mechanisms that govern heat pipe overall thermal resistance, and may be relied upon to develop approximate design guidelines; however, these additional mechanisms should be accounted for in detailed numerical models for application-oriented design and optimization of ultra-thin form factor heat pipes.

\section{REFERENCES}

[1] J.A. Weibel, S.V. Garimella, "Recent Advances in Vapor Chamber Transport Characterization for High-Heat-Flux Applications,” in Advances in Heat Transfer, vol. 45, pp. 209-301, 2013.

[2] S.V. Garimella, C.B. Sobhan, "Recent Advances in the Modeling and Applications of Nonconventional Heat Pipes," in Advances in Heat Transfer, vol. 35, pp. 249-308, 2001.

[3] A. Faghri, "Review and Advances in Heat Pipe Science and Technology,” in Journal of Heat Transfer, vol. 134, p. $123001,2012$.

[4] R.S. Prasher, "A Simplified Conduction Based Modeling Scheme for Design Sensitivity Study of Thermal Solution Utilizing Heat Pipe and Vapor Chamber Technology,” in Journal of Electronic Packaging, vol. 125, pp. 378-385, 2003.

[5] J.-Y. Chang, R.S. Prasher, S. Prstic, P. Cheng, H.B. Ma, "Evaporative Thermal Performance of Vapor Chambers Under Nonuniform Heating Conditions," in Journal of Heat Transfer, vol. 130, p. 121501, 2008.

[6] K. Vafai, W. Wang, "Analysis of Flow and Heat Transfer Characteristics of an Asymmetrical Flat Plate Heat Pipe," in International Journal of Heat and Mass Transfer, vol. 35, pp. 2087-2099, 1992.

[7] Y. Wang, K. Vafai, "Transient Characterization of Flat Plate Heat Pipes During Startup and Shutdown Operations," in International Journal of Heat and Mass Transfer, vol. 43, 2641-2655, 2000.

[8] M. Aghvami, A. Faghri, “Analysis of Flat Heat Pipes with Various Heating and Cooling Configurations,” in Applied Thermal Engineering, vol. 31, pp. 2645-2655, 2011.

[9] Y. Koito, H. Imura, M. Mochizuki, Y. Saito, S. Torii, "Numerical Analysis and Experimental Verification on Thermal Fluid Phenomena in a Vapor Chamber," in Applied Thermal Engineering, vol. 26, pp. 1669-1676, 2006.

[10] B. Xiao, A. Faghri, “A Three-Dimensional Thermal-Fluid Analysis of Flat Heat Pipes,” in International Journal of Heat and Mass Transfer, vol. 51, pp. 3113-3126, 2008.

[11] U. Vadakkan, J.Y. Murthy, S.V. Garimella, “Transient Analysis of Flat Heat Pipes,” Proceedings of the ASME Summer Heat Transfer Conference (SHTC) Las Vegas, NV, USA, July 21-23, 2003.

[12] U. Vadakkan, S.V. Garimella, J.Y. Murthy, "Transport in Flat Heat Pipes at High Heat Fluxes from Multiple Discrete Sources," in Journal of Heat Transfer, vol. 126, pp. 347-354, 2004.

[13] R. Ranjan, J.Y. Murthy, S.V. Garimella, U. Vadakkan, “A Numerical Model for Transport in Flat Heat Pipes Considering Wick Microstructure Effects,” in International Journal of Heat and Mass Transfer, vol. 54, pp. 153-168, 2001.

[14] M. Famouri, G. Carbajal, C. Li, "Transient Analysis of Heat Transfer and Fluid Flow in a Polymer-Based Micro Flat Heat Pipe with Hybrid Wicks," in International Journal of Heat and Mass Transfer, vol. 70, pp. 545-555, 2014.

[15] D. Reay, P. Kew, Heat Pipes: Theory, Design, and Applications, 5th Edition, Butterworth-Heinemann, 2006.

[16] I. Sauciuc, G. Chrysler, R. Mahajan, R. Prasher, “Spreading in the Heat Sink Base: Phase Change Systems or Solid Metals??,” in IEEE Transactions on Components and Packaging Technologies, vol. 25, pp. 621-628, 202.

[17] S.A. Klein, Engineering Equation Solver (EES), F-Chart Software, 1992-2013.

[18] R.W. Schrage, A Theoretical Study of Interface Mass Transfer, Columbia University Press, New York, 1953.

[19] ANSYS FLUENT User's Guide, ANSYS Inc., Release 14.0, (2011). 
[20] K.K. Bodla, J.Y. Murthy, S.V. Garimella, “Direct Simulation of Thermal Transport Through Sintered Wick Microstructures,” in Journal of Heat Transfer, vol. 134, p. 012602, 2011.

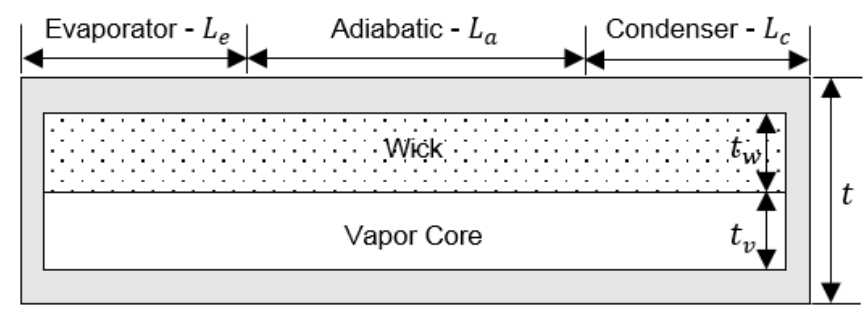

Adiabatic Exterior Walls

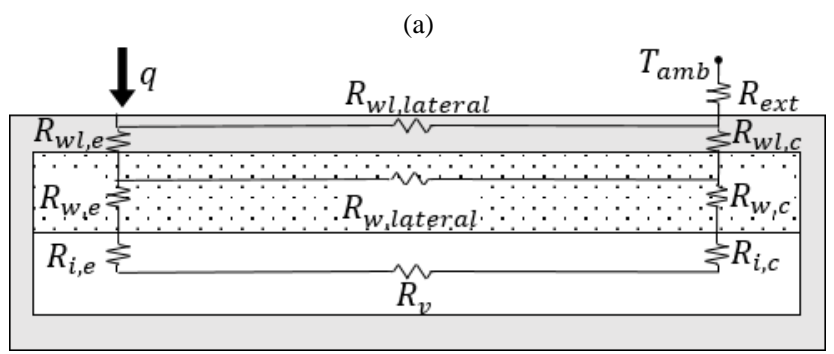

(b)

Fig. 1. Schematic diagrams of (a) the heat pipe geometry under consideration, and (b) the approximate network of thermal resistances between the evaporator and ambient. 


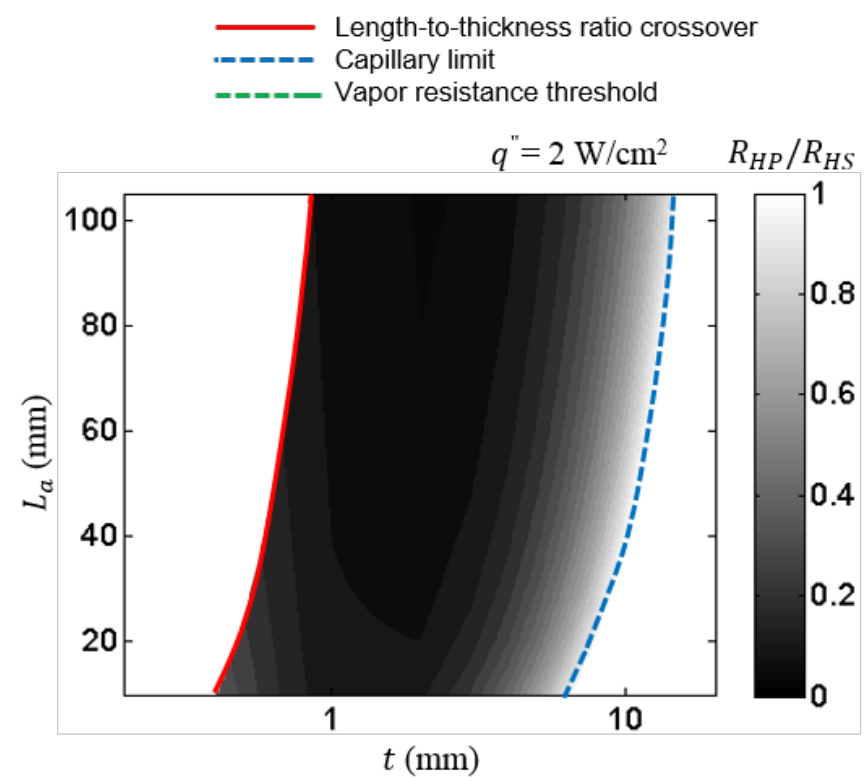

(a)

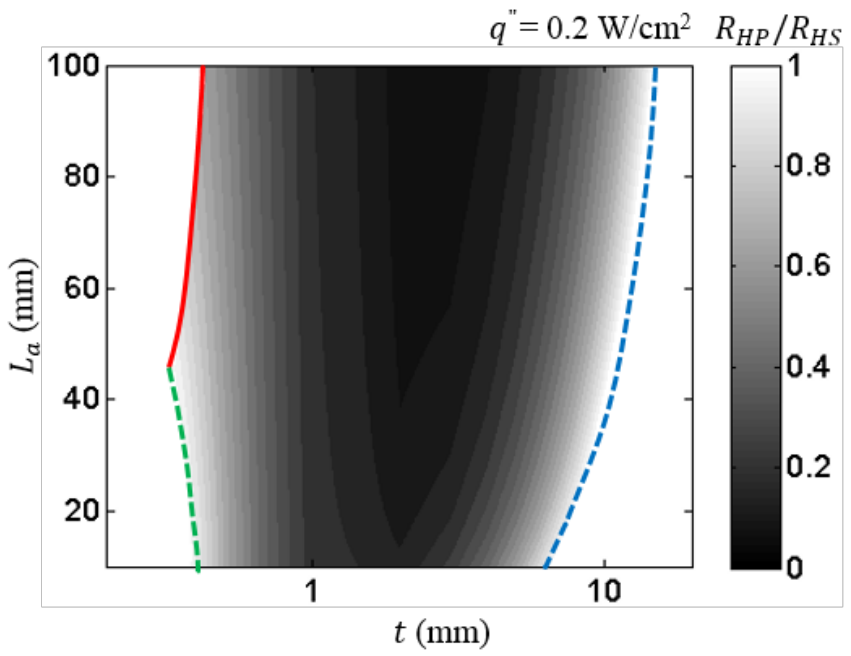

(b)

Fig. 2. Contour map of the resistance ratio $R_{H P} / R_{H S}$ plotted as a function of adiabatic length and thickness for an input heat flux of (a) 2 $\mathrm{W} / \mathrm{cm}^{2}$, and (b) $0.2 \mathrm{~W} / \mathrm{cm}^{2}$. 


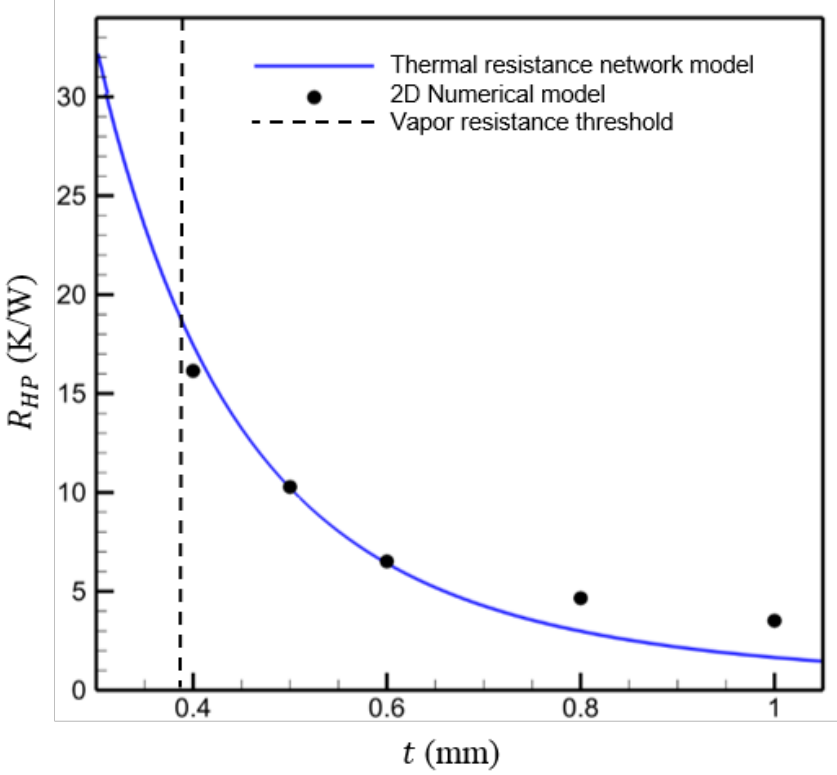

Fig. 3. Comparison of effective thermal resistance of the heat pipe as predicted by the thermal resistance network model and 2D numerical model $\left(\mathrm{L}_{\mathrm{a}}=20 \mathrm{~mm}, q "=0.2 \mathrm{~W} / \mathrm{cm}^{2}\right)$. 


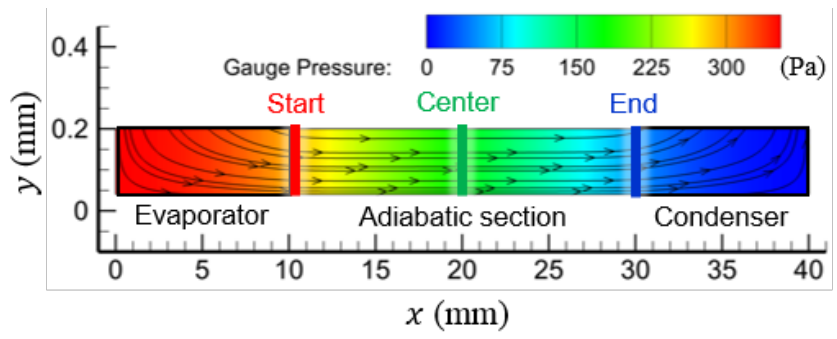

(a)

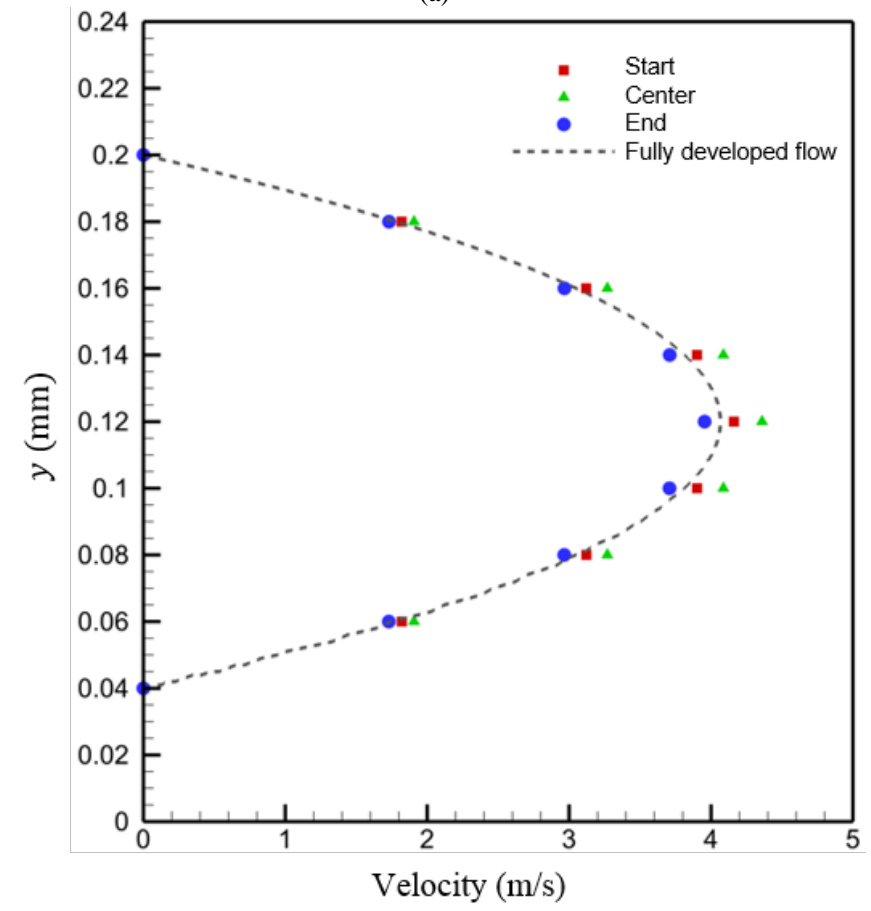

(b)

Fig. 4. (a) Contours of gauge pressure with streamtraces overlaid in the vapor core for a heat pipe of $t=0.4 \mathrm{~mm}$ and $L_{a}=20 \mathrm{~mm}$ and (b) comparison of the velocities predicted by the numerical model across the adiabatic section against a fully developed velocity profile. 


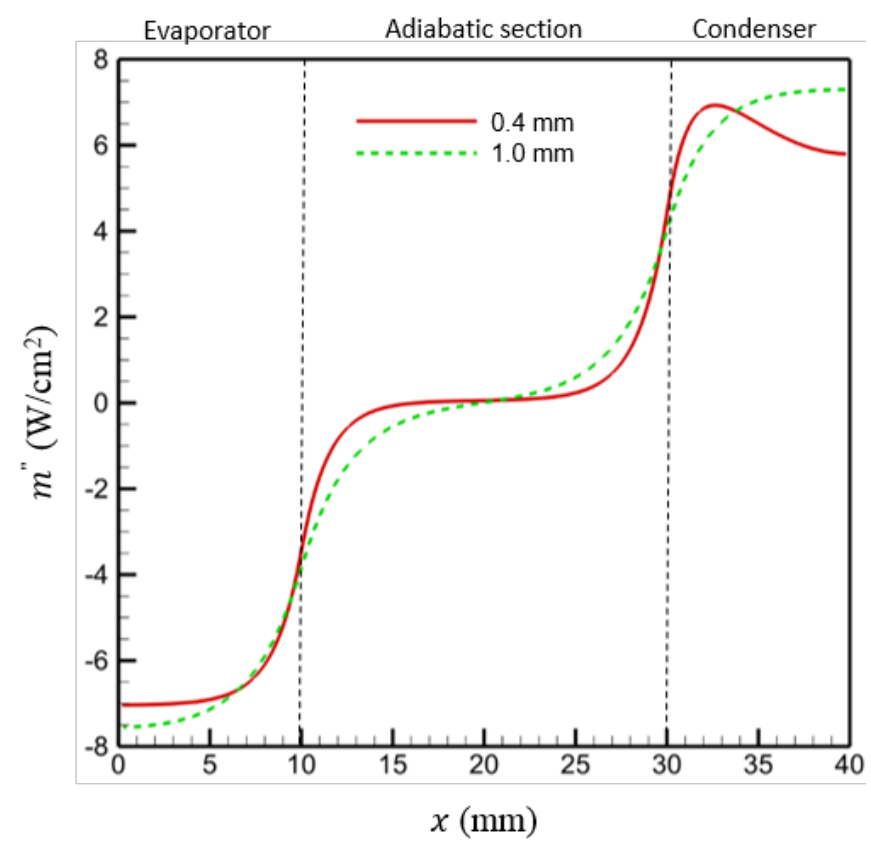

Fig. 5. The mass flux along the wick-vapor interface for a device with $L_{a}=20 \mathrm{~mm}$, and heat input $q^{\prime \prime}=0.2 \mathrm{~W} / \mathrm{cm}^{2}$

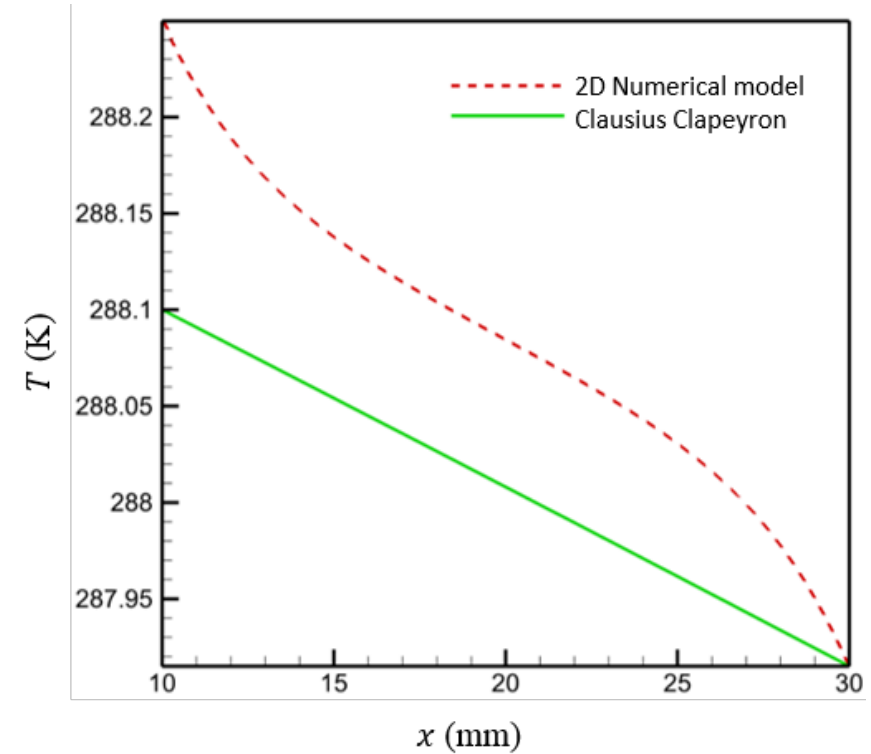

Fig. 6. Temperature variation along the centerline of the adiabatic section for a heat pipe with $t=1.0 \mathrm{~mm}$ and $L_{a}=20 \mathrm{~mm}$. 
TABLE I

COMPARISON OF TEMPERATURE DROP ACROSS THE WICK IN THE EVAPORATOR AND CONDENSER SECTIONS

\begin{tabular}{ccccc}
\hline \hline \multirow{2}{*}{$t$} & \multicolumn{2}{c}{$\Delta T_{\text {wick,evaporator }}$} & \multicolumn{2}{c}{$\Delta T_{\text {wick,condenser }}$} \\
\cline { 2 - 5 }$(\mathrm{mm})$ & $2 \mathrm{D}$ & $1 \mathrm{D}$ & $2 \mathrm{D}$ & $1 \mathrm{D}$ \\
\hline 0.4 & 0.0054 & 0.0050 & 0.0052 & 0.0050 \\
0.5 & 0.0065 & 0.0065 & 0.0062 & 0.0065 \\
0.6 & 0.008 & 0.008 & 0.0078 & 0.008 \\
0.8 & 0.010 & 0.011 & 0.010 & 0.011 \\
1.0 & 0.012 & 0.014 & 0.012 & 0.014 \\
\hline \hline
\end{tabular}

TABLE II

SECTION-WISE COMPARISON OF PRESSURE DROP IN VAPOR CORE

\begin{tabular}{ccccccc}
\hline \hline \multirow{2}{*}{$t$} & \multicolumn{2}{c}{$\Delta P_{\text {evap }}$} & \multicolumn{2}{c}{$\Delta P_{\text {adi }}$} & \multicolumn{2}{c}{$\Delta P_{\text {cond }}$} \\
\cline { 2 - 7 }$(\mathrm{mm})$ & 2D & FD flow & 2D & FD flow & 2D & FD flow \\
\hline 0.4 & 59.0 & 59.1 & 232.9 & 236.0 & 51.8 & 59.1 \\
0.5 & 32.7 & 33.2 & 130.6 & 132.8 & 30.2 & 33.2 \\
0.6 & 19.7 & 20.1 & 79.6 & 80.3 & 17.7 & 20.1 \\
0.8 & 9.4 & 9.8 & 38.2 & 39.0 & 9.0 & 9.8 \\
1.0 & 4.9 & 5.1 & 19.9 & 20.4 & 4.7 & 5.1 \\
\hline \hline
\end{tabular}

TABLE III

COMPARISON OF TEMPERATURE DROP IN THE ADIABATIC SECTION

\begin{tabular}{ccc}
\hline \hline \multirow{2}{*}{$t$} & \multicolumn{2}{c}{$\Delta T_{a d i}$} \\
\cline { 2 - 3 }$(\mathrm{mm})$ & $2 \mathrm{D}$ & $\mathrm{CC}$ \\
\hline 0.4 & 1.84 & 2.06 \\
0.5 & 1.06 & 1.14 \\
0.6 & 0.68 & 0.72 \\
0.8 & 0.38 & 0.33 \\
1.0 & 0.26 & 0.17 \\
\hline \hline
\end{tabular}

